\title{
Improving Context-Awareness in a Healthcare Multi-Agent System
}

\author{
Ricardo S. Alonso, Dante I. Tapia, Óscar García, \\ David Sancho, and Miguel Sánchez
}

\begin{abstract}
Context-aware technologies allow Ambient Assisted Living systems and applications to automatically obtain information from users and their environment in a distributed and ubiquitous way. One of the most important technologies used to provide context-awareness to a system is Wireless Sensor Networks. This paper describes last improvements made on ALZ-MAS, an Ambient Intelligence based multi-agent system aimed at enhancing the assistance and healthcare for Alzheimer patients. In this sense, a new ZigBee platform is used to improve ALZ-MAS. This platform provides the system with new telemonitoring and locating engines that facilitate the integration of context-awareness into it.
\end{abstract}

Keywords: Multi-Agent Systems, Ambient Intelligence, Context-Awareness, Wireless Sensor Networks, Real-Time Locating Systems, e-Healthcare.

\section{Introduction}

Dependence is a permanent situation in which a person needs important assistance from others in order to perform basic daily life activities such as essential mobility, object and people recognition, as well as domestic tasks [2]. There is an ever growing need to supply constant care and support to the disabled and elderly, and the drive to find more effective ways of providing such care has become a major challenge for the scientific community. In this sense, Ambient Intelligence (AmI) is an emerging multidisciplinary area based on ubiquitous computing, which

Ricardo S. Alonso · Dante I. Tapia · Óscar García · David Sancho

Nebusens, S.L. Scientific Park of the University of Salamanca, Calle Adaja, s/n, 37185,

Villamayor de la Armuña, Salamanca, Spain.

e-mail: \{ricardo.alonso, dante.tapia, oscar.garcia,

david.sancho\} @nebusens.com

Miguel Sánchez

Pontifical University of Salamanca, Calle Compañía, 5, 37002, Salamanca, Spain

J.M. Corchado et al. (Eds.): Trends in PAAMS, AISC 90, pp. 1-8.

springerlink.com $\quad$ (C) Springer-Verlag Berlin Heidelberg 2011 
influences the design of protocols, communications, systems and devices, proposing new ways of interaction between people and technology, thus adapting them to the needs of individuals and their environment [13].

One key aspect in any AmI-based system is the use of context-aware technologies. The context is defined as any information used to characterize the situation of an entity, which can be a person, a place or an object [3]. This information is important for defining the interaction between users and the technology that surrounds them. However, it is not enough to gather information about the context, but that information must be processed by self-adaptable and dynamic mechanisms and methods that can react independently of each particular situation that arises. In this sense, Multi-Agent Systems (MAS) [14] comprise one of the areas that can contribute expanding the possibilities of Ambient Assisted Living [8]. Furthermore, most of the context information can be collected by distributed sensors throughout the environment and even by the users themselves. Wireless Sensor Networks (WSN), such as ZigBee [15], are more flexible and require less infrastructural support than wired sensor networks [9], avoiding the need of wiring homes or hospitals and decreasing the costs and drawbacks of the setup phase.

This paper describes the new changes introduced into ALZ-MAS [1], an Ambient Intelligence based multi-agent system aimed at enhancing the assistance and healthcare for Alzheimer patients in geriatric residences. An essential aspect in this work is the use of Wireless Sensor Networks to provide the agents with automatic and real-time information of the environment and allow them to react upon it. Therefore, these new changes include the integration of n-Core [7], an innovative wireless sensor platform, to improve the context-awareness of the system with better and new telemonitoring and locating techniques.

The problem description that motivated the development of ALZ-MAS is presented in the next section. Then, it is described the basic components of ALZMAS and the new changes introduced in the system to provide its agents with improved context-aware capabilities. In addition, it is explained some experiments made to evaluate the performance of the system applied to a real scenario, as well as the obtained results, comparing the previous version of ALZ-MAS and the new release presented in this paper. Finally, conclusions are depicted.

\section{Problem Description}

Ambient Intelligence offers a great potential to improve quality of life and simplify the use of technology by offering a wider range of personalized services and providing users with easier and more efficient ways to communicate and interact with other people and systems [13]. Among the general population, those most likely to benefit from the development of these systems are the elderly and dependent people, whose daily lives, with particular regard to healthcare, will be most enhanced [11]. Dependent people can suffer from degenerative diseases, dementia, or loss of cognitive ability [2]. However, the development of systems that clearly fulfill the needs of AmI is difficult and not always satisfactory. It requires a joint development of models, techniques and technologies based on services. 
As mentioned before, one of the key aspects for the construction of AmI-based systems is obtaining information from the context through sensor networks. The context includes information about people and their environment. The information may consist of many different parameters such as location, building status (e.g., temperature), vital signs (e.g., heart rhythm), etc.

Sensor networks are presented as an optimal technology for obtaining contextual information. Wired sensor networks, such as X10, LonWorks or KNX, are not as flexible as wireless sensors networks, such as ZigBee, and require more infrastructural support [9]. The ZigBee standard [15] operates in the $868 / 915 \mathrm{MHz}$ and $2.4 \mathrm{GHz}$ unlicensed bands. ZigBee is designed to work with low-power nodes and allows up to 65,534 nodes to be connected in a star, tree or mesh topology network. There are several telemonitoring healthcare developments based on Wireless Sensor Networks [6, 12]. However, these developments do not take into account their integration with other systems or architectures and are difficult to be adapted to other different scenarios.

Furthermore, the information obtained must be managed by intelligent and selfadaptable technologies to provide an adequate interaction between the users and their environment. Agents and Multi-Agent Systems are one of these technologies. Agents have a set of characteristics, such as autonomy, reasoning, reactivity, social abilities, pro-activity, mobility or organization which allow them to cover several needs for Ambient Assisted Living environments, as they possess the capability of adapting themselves to the users and environmental characteristics [5]. Multi-agent systems and architectures based on intelligent devices have recently been used to provide assistance to elderly and dependent people [8]. Although these applications expand the possibilities and stimulate research efforts to enhance the assistance and healthcare provided to elderly and dependent people, none of them integrate intelligent agents, distributed and dynamic applications and services approach or the use of reasoning and planning mechanisms.

\section{ALZ-MAS: An Alzheimer Healthcare Multi-Agent System}

ALZ-MAS (ALZheimer Multi-Agent System) $[1,10]$ is a distributed multi-agent system designed upon Ambient Intelligence and aimed at enhancing the assistance and healthcare for Alzheimer patients living in geriatric residences. The main functionalities in the system include reasoning and planning mechanisms [4] that are embedded into deliberative BDI (Belief-Desire-Intention) agents, and the use of several context-aware technologies to acquire information from users and their environment. The current version of the system, ALZ-MAS 2.0, makes use of FUSION@ (Flexible User and ServIces Oriented multiageNt Architecture), an architecture that combines a SOA approach with intelligent agents for building highly dynamic Ambient Intelligence based systems [10].

As can be seen in Figure 1, ALZ-MAS structure has five different deliberative agents based on the BDI model (BDI Agents), each one with specific roles and capabilities: User Agent, SuperUser Agent, ScheduleUser Agent, Admin Agent and Devices Agent. In ALZ-MAS, each agent integrates its own functionalities into their structure. If an agent needs to perform a task which involves another agent, it 
must communicate with that agent to request it. So, if the agent is disengaged, all its functionalities will be unavailable to the rest of agents. Another important issue is that the reasoning (CBR) and planning (CBP) mechanisms are integrated into the agents. The main problem with ALZ-MAS 1.0 was the efficiency of the computational planning processes as well as the integration with other existing architectures. This way, the main components of ALZ-MAS 2.0 were modeled as distributed and independent services by means of FUSION@, releasing the agents from high demanding computational processes. FUSION@ proposes a new perspective, where Multi-Agent Systems and SOA-based services are integrated to provide ubiquitous computation, ubiquitous communication and intelligent interfaces facilities. As can be seen in Figure 1, the FUSION@ architecture defines four basic blocks: Applications, Services, Agents Platform and Communication Protocol [10]. Besides, there are seven pre-defined agents that provide the basic functionalities of the architecture: CommApp, CommServ, Directory, Supervisor, Security, Admin and Interface. Interface Agents were designed to be embedded in users' applications and are simple enough to allow them to be executed on mobile devices, such as cell phones or PDAs.

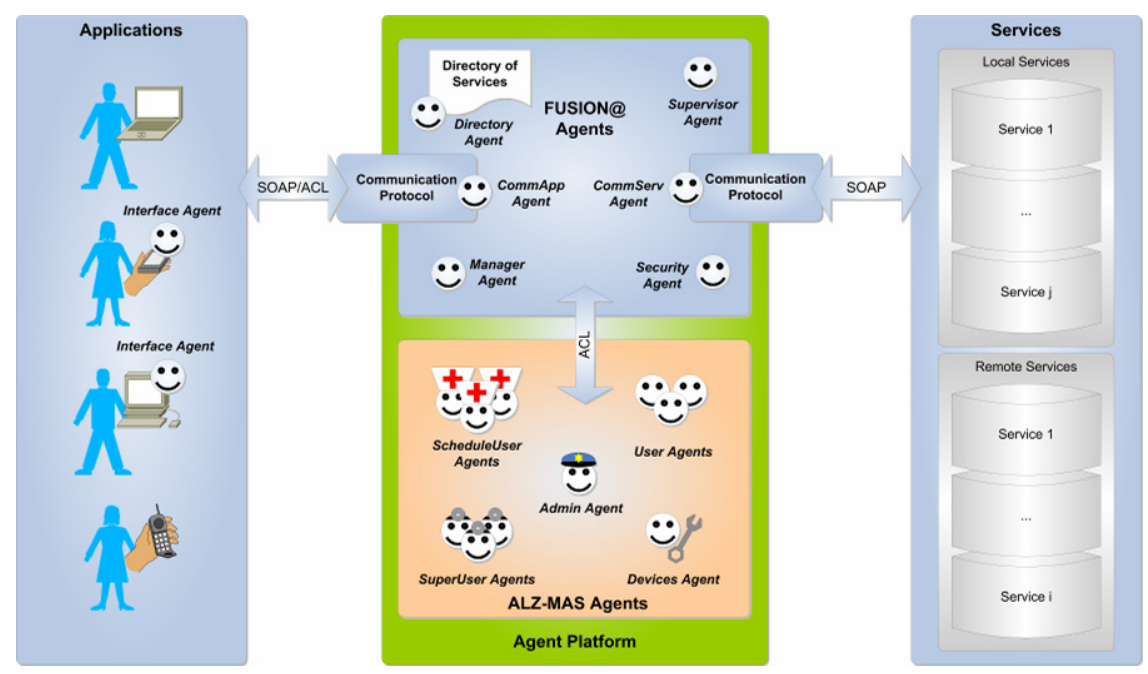

Fig. 1 Schema of the ALZ-MAS 2.0 system

Agents in ALZ-MAS 2.0 [10] collaborate with context-aware agents that employ Radio Frequency IDentification (RFID) [1], Wireless Sensor Networks and automation devices to provide automatic and real-time information about the environment, and allow users to interact with their surroundings, controlling and managing physical services (i.e., heating, lights, switches, etc.). All the information provided is processed by the agents, specially the Devices Agent which is a BDI agent that runs on a workstation. All hardware is someway integrated with agents, 
providing automatic and real-time information about the environment that is processed by the agents to automate tasks and manage multiple services.

The configuration used in ALZ-MAS 2.0 consisted of an RFID transponder mounted on a bracelet worn on the users' wrist or ankle, several RFID readers installed over protected zones with an adjustable capture range up to 2 meters, and a central workstation where all the information is processed and stored. These RFID readers were installed over the doors accessing to restricted zones so that the system could detect when a user was trying to enter a forbidden area according to the user's permissions. Nevertheless, this configuration does not allow knowing the exact position of each user in real-time. Moreover, this approach requires gathering information from two distinct sensor networks: an RFID network for detecting unauthorized accesses and other ZigBee network to obtain information of the environment from different sensors, such as temperature sensors, light sensors, as well as smoke and gas detectors. Thus, the new ALZ-MAS 2.1 has been improved with the integration of an innovative ZigBee platform into the system to provide it with enhanced telemonitoring and new locating features.

\subsection{Improving the Context-Aware Technologies Used in ALZ-MAS 2.0}

As mentioned before, ALZ-MAS 2.0 used a combination of RFID and ZigBee technologies to obtain context information. This way, while RFID tags and readers was used to allow the system to detect unauthorized accesses to restricted areas, a ZigBee network was used to provide the system with sensing capacities. Nevertheless, the new ALZ-MAS 2.1 uses just ZigBee devices to obtain both users' location and other context information.

Figure 2 (left) shows the ZigBee devices used in ALZ-MAS 2.1 to control heating, lights, door locks, alarms, etc. These devices, called n-Core Sirius-A, have both $2.4 \mathrm{GHz}$ and $868 / 915 \mathrm{MHz}$ versions and have several communication ports (GPIO, ADC, I2C and UART through USB or DB-9 RS-232) to connect to distinct devices, including a wide range of sensors and actuators [7]. This way, it is deployed a mesh of these devices to control all these services. n-Core Sirius-A devices are part of the n-Core platform (http://www.n-core.info), which offers a complete API (Application Programming Interface) to access all its functionalities, including telemonitoring and locating engines. This API can be easily accessed as a Dynamic Link Library (DLL) from different languages and platforms, such as $\mathrm{C} / \mathrm{C}++$, .Net, Java or Python. Thus, it can be used different locating techniques using Sirius A devices as readers and Sirius B devices, showed in Fig. 2 (right), as tags carried by patients and medical personnel. Sirius B devices are small enough to be carried by a patient, a caregiver or even an object, and provide a battery life of up to two years. 

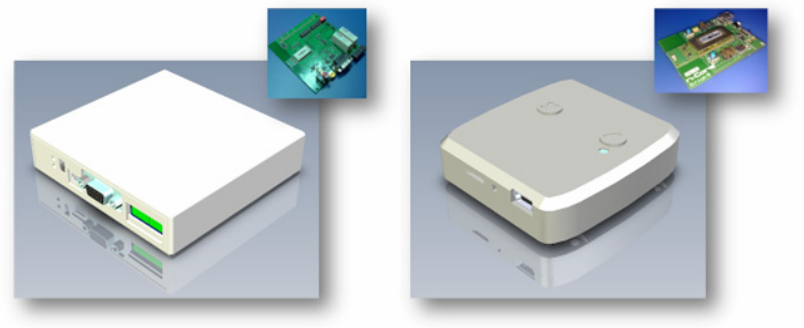

Fig. 2 n-Core Sirius A (left) and Sirius B (right) devices used in ALZ-MAS 2.1

In ALZ-MAS 2.0, the system could only know in which room each user was, because location was based on knowing which door (i.e., RFID antenna) a user was passed through. In ALZ-MAS 2.1 the location of users is given as coordinated points obtained from the locating techniques provided by the n-Core API. All information obtained by means of these technologies is processed by the agents. Nonetheless, thanks to the architecture of the system, it was not necessary to introduce any change into the agents' reasoning mechanisms. Depending on the system requirements, several interfaces can be executed. The interfaces show basic information about nurses and patients (name, tasks that must be accomplished, location inside the residence, etc.) and the building (specific room temperature, lights status, etc.). Figure 3 shows one of the new user interfaces of ALZ-MAS 2.1., where it is shown the positions of the users given by the new locating engine.

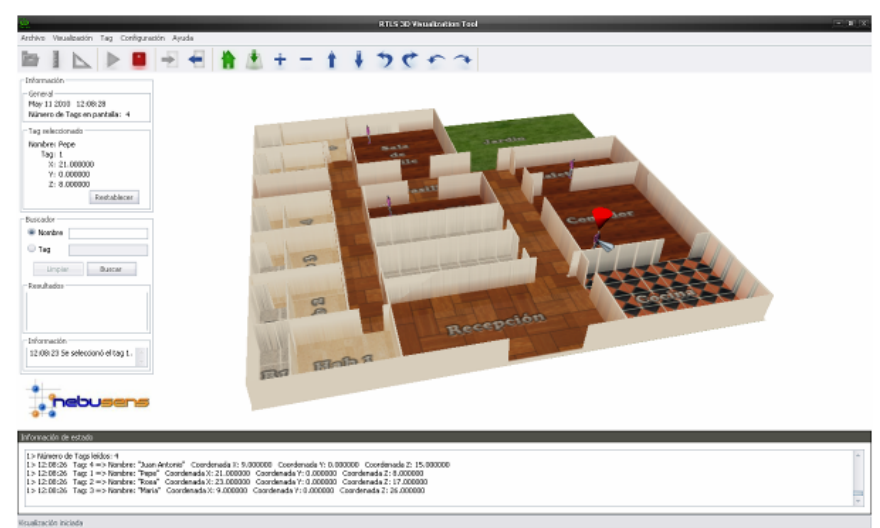

Fig. 3 Graphical User Interface of the ALZ-MAS 2.1

After the inclusion of n-Core into ALZ-MAS, it has been done new experiments to test the improvements made in the context-awareness of ALZ-MAS. This way, the new ALZ-MAS 2.1 prototype using new n-Core ZigBee devices and API [7] for both locating and telemonitoring tasks was implemented in the same center [1] and compared with previous ALZ-MAS 2.0, using both RFID and ZigBee devices. One of these experiments consisted of testing the performance of 
ALZ-MAS when detecting users accessing to restricted areas according to their permissions. ALZ-MAS monitors and locate the patients and guarantees that each one of them is in the right place, and secondly, only authorized personnel can gain access to the residence protected areas. Figure 4 shows the number of accesses to restricted zones detected before the implementation of ALZ-MAS 2.1 with the previous release of ALZ-MAS 2.0 during a 30-day period. With the new release of ALZ-MAS 2.1 using new ZigBee devices and more accurate locating techniques, there is an improvement regarding the previous ALZ-MAS version, increasing from 3 to 8 daily detections. Furthermore, thanks to the new innovative locating techniques, the number of daily false positives has been reduced from 7 to 0.87 .

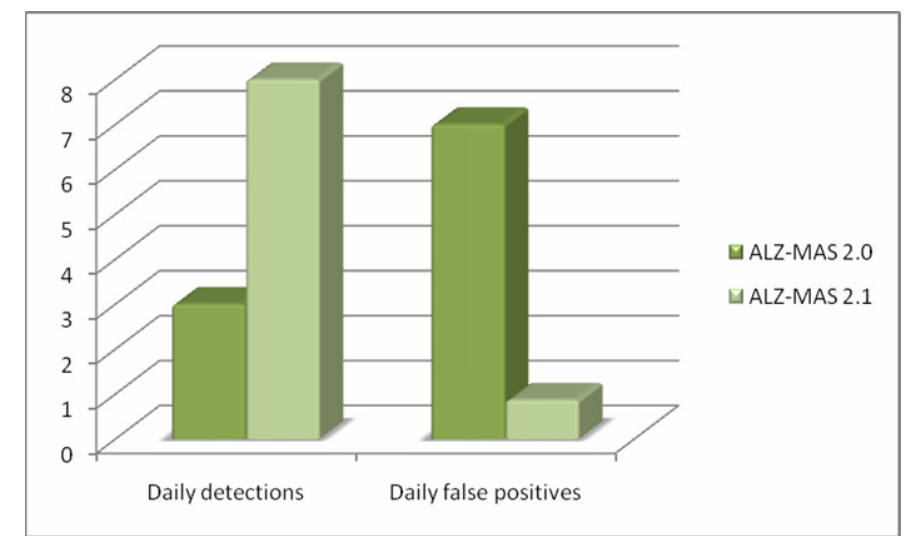

Fig. 4 Comparison of the daily number of detected accesses to restricted areas and false positives between ALZ-MAS 2.0 and ALZ-MAS 2.1

\section{Conclusions}

ALZ-MAS can improve the security and healthcare efficiency through monitoring medical staff's work and patients' activities. Context-aware technologies provide ALZ-MAS with valuable information about the environment, contributing to a ubiquitous and non-invasive interaction among users, the system and the environment. Thanks to the n-Core platform, the new ALZ-MAS 2.1 improves significantly the context information in respect of ALZ-MAS 2.0. Qualitatively, the location information is provided as coordinated positions, instead of just which room each user is. Quantitatively, security has been clearly improved. Furthermore, infrastructure costs have been reduced in ALZ-MAS 2.1 in relation to ALZ-MAS 2.0. Although each RFID transponder is cheaper than a ZigBee device used as tag, RFID antennas are quite more expensive than ZigBee devices used as readers. Even more, to obtain the same level of precision in the location information than the provided by the ZigBee platform, it would be necessary many more expensive RFID readers. 
Acknowledgments. This This research has been partially supported by the project PET2008_0036 and FEDER funds.

\section{References}

1. Corchado, J.M., Bajo, J., Paz, Y.D., Tapia, D.I.: Intelligent environment for monitoring Alzheimer patients, agent technology for health care. Decis. Support Syst. 44(2), 382-396 (2008)

2. Costa-Font, J., Patxot, C.: The design of the long-term care system in Spain: Policy and financial constraints. Social Policy and Society 4(1), 11-20 (2005)

3. Dey, A.K., Abowd, G.D.: Towards a Better Understanding of Context and ContextAwareness. In: Proceedings of the CHI 2000 Workshop on The What, Who, Where, When, and How of Context-Awareness. The Hague, Netherlands (2000)

4. Glez-Bedia, M., Corchado, J.M.: A planning Strategy based on Variational Calculus for Deliberative Agents. Computing and Information Systems Journal 10, 2-14 (2002)

5. Jayaputera, G.T., Zaslavsky, A.B., Loke, S.W.: Enabling run-time composition and support for heterogeneous pervasive multi-agent systems. Journal of Systems and Software 80(12), 2039-2062 (2007)

6. Jurik, A.D., Weaver, A.C.: Remote Medical Monitoring. Computer 41(4), 96-99 (2008)

7. n-Core: A Faster and Easier Way to Create Wireless Sensor Networks (2010), http: / /www.n-core.info (retrieved January 9, 2011)

8. Pecora, F., Cesta, A.: Dcop for smart homes: A case study. Computational Intelligence 23(4), 395-419 (2007)

9. Sarangapani, J.: Wireless Ad hoc and Sensor Networks: Protocols, Performance and Control. CRC, Boca Raton (2007)

10. Tapia, D.I., Bajo, J., Corchado, J.M.: Distributing Functionalities in a SOA-Based Multi-agent Architecture. In: 7th International Conference on Practical Applications of Agents and Multi-Agent Systems (PAAMS 2009), pp. 20-29 (2009)

11. Van Woerden, K.: Mainstream Developments in ICT: Why are They Important for Assistive Technology? Technology and Disability 18(1), 15-18 (2006)

12. Varshney, U.: Improving Wireless Health Monitoring Using Incentive-Based Router Cooperation. Computer 41(5), 56-62 (2008)

13. Weber, W., Rabaey, J.M., Aarts, E.: Ambient Intelligence. Springer-Verlag New York, Inc., Heidelberg (2005)

14. Wooldridge, M.: An Introduction to MultiAgent Systems. John Wiley \& Sons, Chichester (2002)

15. ZigBee Alliance, ZigBee Specification Document 053474r13. ZigBee Standards Organization (2006) 